\title{
Fermentación alcohólica: Una opción para la producción de energía renovable a partir de desechos agrícolas
}

\section{Alcoholic fermentation: An option for renewable energy production from agricultural residues}

\author{
H.J. Vázquez ${ }^{1}$ y O. Dacosta ${ }^{2}$ \\ ${ }^{1}$ Departamento de Sistemas, Universidad Autónoma Metropolitana, Unidad Azcapotzalco, México y \\ Oficina de Consejo, Desarrollo y Transferencia Tecnológica, Dijon, Francia \\ E-mails: hjv@correo.azc.uam.mx,statfor@yahoo.com
}

(Recibido: abril de 2006; aceptado: mayo de 2007)

\begin{abstract}
Resumen
La biotecnología ofrece diversas opciones para la generación de energías renovables. Una de ellas es la producción de bioetanol, el cual se obtiene mediante fermentación. El bioetanol se usa en la preparación de carburantes para vehículos automotores. En este artículo se presenta una propuesta para la obtención de este combustible mediante una unidad de fermentación piloto exper i mental de 100 litros. Los resultados de nuestros ensayos, en rendimiento y productividad, son similares a los de otros laboratorios si se considera que esta unidad piloto funciona en condiciones no estériles, lo que representa como ventaja un ahorro de energía no despreciable. Además, la tecnología no requiere conocimientos especializados para su realización y estaría al alcance de grupos campesinos mexicanos.
\end{abstract}

Descriptores: Biotecnología, alcohol, energías renovables, vehículos.

\begin{abstract}
Biotechnology offers severaloptionsforgenerat ing renewableenergy. Oneof thesetechnol ogies con sists on produc ing bioethanol by fer men ta tion. Bioethanol is manly used to pre pare fuel for mo tor ve hicles. This pa per pres ents a pro posal to pro duce such as fu els withahundredlitersexperimen talfermen tation pilot unit. Resultsderived fromessays are sim i lar, in terms of yield and pro duc tiv ity, to those pre sented by other sys tems, if we take into ac count that our unit works un der non ster ile con di tions, which rep re sents sig nificanten ergy sav ings. This tech nol ogy does not re quire specialized knowl edge for its con struc tion and it would accesible to groups of mex i can farmers.
\end{abstract}

Key words: Biotechnology, alcohol, energiesrenewable, vehicles.

\section{Introducción}

En los últimos años, en México se ha destacado la urgencia de realizar reformas estructurales que permitan un mayor desarrollo para enfrentar las necesidades que la globalización que la economía trae consigo. El sector energético es uno de los campos en los que se resalta la importancia de efectuar cambios y mejoras. Sin embargo, por ser un bien de interés público, las dificultades de cambio se explican por la gran rigidez en las políticas y reglamentos que regulan los procesos de producción, almacenamiento, transporte y distribución de energía; principalmente de energías no renovables. Sin 
Fermentación alcohólica: Una opción para la producción de energía renovable a partir de ...

embargo, es importante buscar otras soluciones orientadas más al desarrollo de sistemas eficientes en cuanto al consumo de energía y a la búsqueda de nuevas fuentes de energía, que limitarse al cambio de políticas y reglamentos de explotación, de lo que queda, de las fuentes de energía no renovables existentes (Maurice, 2007).

En general, las fuentes de energía, se clasifican como renovables y no renovables. Entre las primeras se encuentra la energía eólica, hidráulica, geotérmica, maremotriz, solar y las energías de la biomasa (bioetanol, combustión directa de biomasa leñosa, combustión con gasificación, la pirolisis y la producción de gas por biodigestión anaeróbica.). Dentro de las energías no renovables se encuentra el petróleo, el gas natural (metano) y la energía nuclear; siendo el petróleo la principal fuente de energía en México, a diferencia de la nuclear, cuya producción es muy limitada, pues cuenta con una potencia efectiva instalada de 1,365 MW.

En lo que respecta a las energías renovables, en México existe potencial para el desarrollo de fuentes de energía de esta naturaleza (Secretaría de Energía, 2005). En el plan publicado por la Secretaría de Energía (SENER) se proporcionan los siguientes datos sobre la capacidad que México tiene en tal aspecto:

- Energías de la biomasa generadas a partir de residuos (desechos sólidos municipales, residuos agropecuarios, de bosques, etcétera) o a partir de biogás, producido por fermentación anaeróbica en plantas de tratamiento de aguas. La cantidad de residuos sólidos municipales se estima en el país en 90,000 toneladas diarias, con lo que se podrían generar aproximadamente $150 \mathrm{MW}$.

- La capacidad potencial de las fuentes de energía hidráulica se evalúan superiores a $3000 \mathrm{MW}$. En cuanto a la energía llamada "mini-hidráulica" se estima que existe potencial para lograr una capacidad media de $400 \mathrm{MW}$.

- En lo que respecta a la generación de electricidad a partir de energía solar, la potencia instalada de sistemas fotovoltaicos se incrementó de 7.1 MW en 1993 a 13 MW en 2000, representando en promedio una tasa de crecimiento anual de 9.3\%. Para el año 2012, se esperan en el país $30 \mathrm{MW}$ instalados y 18 GWh/año de energía producida.

- En materia de energía eólica o energía cinética del viento no existe una evaluación precisa del potencial que hay en el país; sin embargo, se estima supe rior a los 5,000 MW.

- Finalmente, con relación a la energía geotérmica se valora un potencial de 2,400 MW.

Cabe señalar que en el plan publicado por la Secretaría de Energía, consideran energías maduras la hidroelectricidad y la geotermia, las cuales, en conjunto, representaron $25.4 \%$ de la capacidad del Sistema Eléctrico Nacional en 2002.

Una de las fuentes de energía que poco se menciona en los proyectos nacionales y que ha demostrado su factibilidad en otras regiones del mundo, es la producción de etanol. Desde el punto de vista industrial, trabajar en la producción de dicho producto es fundamental, pues el etanol no sólo es una fuente de energía sino una materia prima importante en la industria (química, farmacéutica, agroalimentaria, etc.)

Entre los principales usos del etanol está la preparación de carburantes para vehículos automotores. El etanol permite un aumento 
del índice de octano, y por lo tanto, la reducción del consumo y reducción de la contaminación (10 a 15 \% menos de monóxido de carbono e hidrocarburos). El etanol se puede mezclar con la gasolina sin plomo de un $10 \%$ a un $25 \%$ sin dificultad. En ciertos motores, se ha logrado incorporar hasta en un $100 \%$ (Verdesio, 2003), (Almeida, 2006). El etanol podría así, sustituir al metil ter-butil éter (MTBE), producto oxigenante con el que se reformulan las gasolinas en México desde 1989, y que ha permitido reducir las emisiones de CO2. Esta acción es muy importante pues el MTBE, por ser un compuesto muy estable, de baja degradación y muy soluble en agua, ha resultado ser un contaminante de aguas subterráneas (Nava, 2006). Por otro lado, en algunas regiones, su uso ha disminuido por los riesgos que éste puede representar para la salud, ya que ha sido clasificado como potencialmente carcinógeno, (Lemire, 2004), (EPA, 2007).

Una de las opciones para producir etanol es por fermentación a partir de materias primas ricas en carbohidratos (azúcar, almidón, celulosa, etcétera). Por tal razón, es común designar al etanol obtenido por esta vía "bioetanol". Entre estas materias primas se encuentran las frutas y vegetales como la caña de azúcar y la remolacha, los cereales (trigo, maíz, sorgo), los tubérculos (papas, yuca) y en general, materias provenientes de ligno-celulosas o de residuos orgánicos.

El primer programa masivo de energías renovables a nivel mundial de producción de bioetanol a partir de caña de azúcar, se inició en Brasil en 1975 (Verdesio, 2003). Hasta 1989, el programa se apoyó en políticas públicas de beneficios económicos e incentivos fiscales para ayudar al desarrollo industrial de esta tecnología. La producción se estabilizó desde entonces en 11 a 16 mil millones de litros por año. Sin embargo, al reducirse los subsidios y debido al impor- tante desarrollo del país, tal programa no ha logrado ser suficiente ni ha podido responder a las grandes demandas de energía en Brasil; lo cual no significa que el proyecto haya fracasado, ya que las importaciones de energía hubiesen sido mucho más importantes sin la producción del bioetanol (Almeida, 2006) .

Programas similares se han iniciado en Austria, Canadá, Francia, Alemania, España, Suecia y Estados Unidos. En este último el bioetanol se obtiene a partir del maíz (Coalición Americana por el Etanol, 2006) y en Francia a partir de la remolacha y del trigo (Instituto Francés del Petróleo, 2006). Ahí se estima un costo de producción de bioetanol de 0.38 euro/itro y de 18 euros por Gigajoule. La productividad se estima entre 70 y 75 hectolitros de etanol por hectárea cultivada con remolacha y alrededor de 35 hectolitros de etanol por hectárea cultivada con trigo. El etanol aporta aproximadamente 28 megajoules por kilo (una tonelada de bioetanol equivale aproximadamente a un volumen de 1.25 metros cúbicos, es decir, 12.5 hectolitros). En Francia, el consumo llega apenas al 1\%, pero se estima que llegará al $5.75 \%$ para el año 2010.

Según la Agencia Internacional de Energía (AIE, 2006), el potencial de esta fuente de energía es considerable, pues se calcula que el bioetanol podría sustituir un $25 \%$ de la gasolina utilizada como combustible en el año 2025.

Con los datos preliminares aquí expuestos, es necesario apuntar que el objetivo del presente escrito es presentar los principios que se han utilizado para producir etanol por fermentación alcohólica a partir de melazas $^{1}$ y los resultados de un sistema pi-

1 Las melazas son desechos de las industrias de producción de azúcar a partir de la remolacha. 
Fermentación alcohólica: Una opción para la producción de energía renovable a partir de ...

loto, en el cual participó el autor (Vázquez, 1993), diseñado en la Estación de Microbiología, hoy la Plataforma de Predesarrollo en Biotecnología (PPB) en Dijón, Francia. Es un proyecto en el que la tecnología es relativamente simple y es la base de desarrollo de instalaciones reales y operacionales con capacidad para producir hasta $300.000 \mathrm{hl} / \mathrm{año}$ (Instituto Francés del Petróleo, 2006). Su impulso en México parece factible, además que representaría una oportunidad para diversificar las actividades de producción energética y agrícola del país; y una oportunidad para el desarrollo del campo.

\section{La fermentación alcohólica}

La fermentación alcohólica es una bioreacción que permite degradar azúcares en alcohol y dióxido de carbono. La conversión se representa mediante la ecuación:

$$
\mathrm{C}_{6} \mathrm{H}_{12} \mathrm{O}_{6} \rightarrow 2 \mathrm{C}_{2} \mathrm{H}_{5} \mathrm{OH}+2 \mathrm{CO}_{2}
$$

Las principales responsables de esta transformación son las levaduras. La Saccharomyces cerevisiae, es la especie de levadura usada con más frecuencia. Por supuesto que existen estudios para producir alcohol con otros hongos y bacterias, como la Zymomonas mobilis, pero la explotación a nivel industrial es mínima.

A pesar de parecer, a nivel estequiométrico, una transformación simple, la secuencia de transformaciones para degradar la glucosa hasta dos moléculas de alcohol y dos moléculas de bióxido de carbono es un proceso muy complejo, pues al mismo tiempo la levadura utiliza la glucosa y nutrientes adi cionales para reproducirse. Para evaluar esta transformación, se usa el rendimiento biomasa/producto y el rendimiento producto/substrato.

- Rendimiento biomasa/substrato (Yx/s): es la cantidad de levadura producida por cantidad de substrato consumido.
- Rendimiento substrato/producto ( $\mathrm{Yp} / \mathrm{s})$ : es la cantidad de producto sintetizado por cantidad de substrato consumido.

El rendimiento teórico estequiométrico para la transformación de glucosa en etanol es de $0.511 \mathrm{~g}$ de etanol y $0.489 \mathrm{~g}$ de $\mathrm{CO}_{2}$ por 1 $\mathrm{g}$ de glucosa. Este valor fue cuantificado por Gay Lussac. En la realidad es difícil lograr este rendimiento, porque como se señaló anteriormente, la levadura utiliza la glucosa para la producción de otros metabolitos. El rendimiento experimental varía entre $90 \%$ y 95\% del teórico, es decir, de 0.469 a $0.485 \mathrm{~g} / \mathrm{g}$. Los rendimientos en la industria varían entre 87 y 93\% del rendimiento teórico (Boudarel, 1984). Otro parámetro importante es la productividad $(\mathrm{g} / \mathrm{h} / \mathrm{h})$, la cual se define como la cantidad de etanol producido por unidad de tiempo y de volumen.

Los parámetros aquí mencionados se definen con relación a la fase y al modo de funcionamiento del bioreactor o fermentador (Figura 1). Por lo general, un bioreactor es un recipiente cilíndrico de doble pared, de vidrio o de acero inoxidable (para el control de la temperatura y esterilización en línea), cubierto de una platina de acero inoxidable. La platina está dotada de entradas y salidas que permiten agregar substratos, nutrientes y substancias como ácidos o bases, extraer productos, o bien, hacer mediciones en línea. La platina permite acoplar un sistema de agitación para mantener la homogeneidad y facilitar, en su caso, la transferencia de oxígeno y nutrientes.

El Bioreactor es el elemento central para la realización de la fermentación alcohólica. Existen diversas opciones para disponer de esta tecnología; por ejemplo, construir una instalación simple (Make your own fuel, 2006), hasta la adquisición de una instalación completa con especificaciones técnicas adecuadas a las características concretas del 


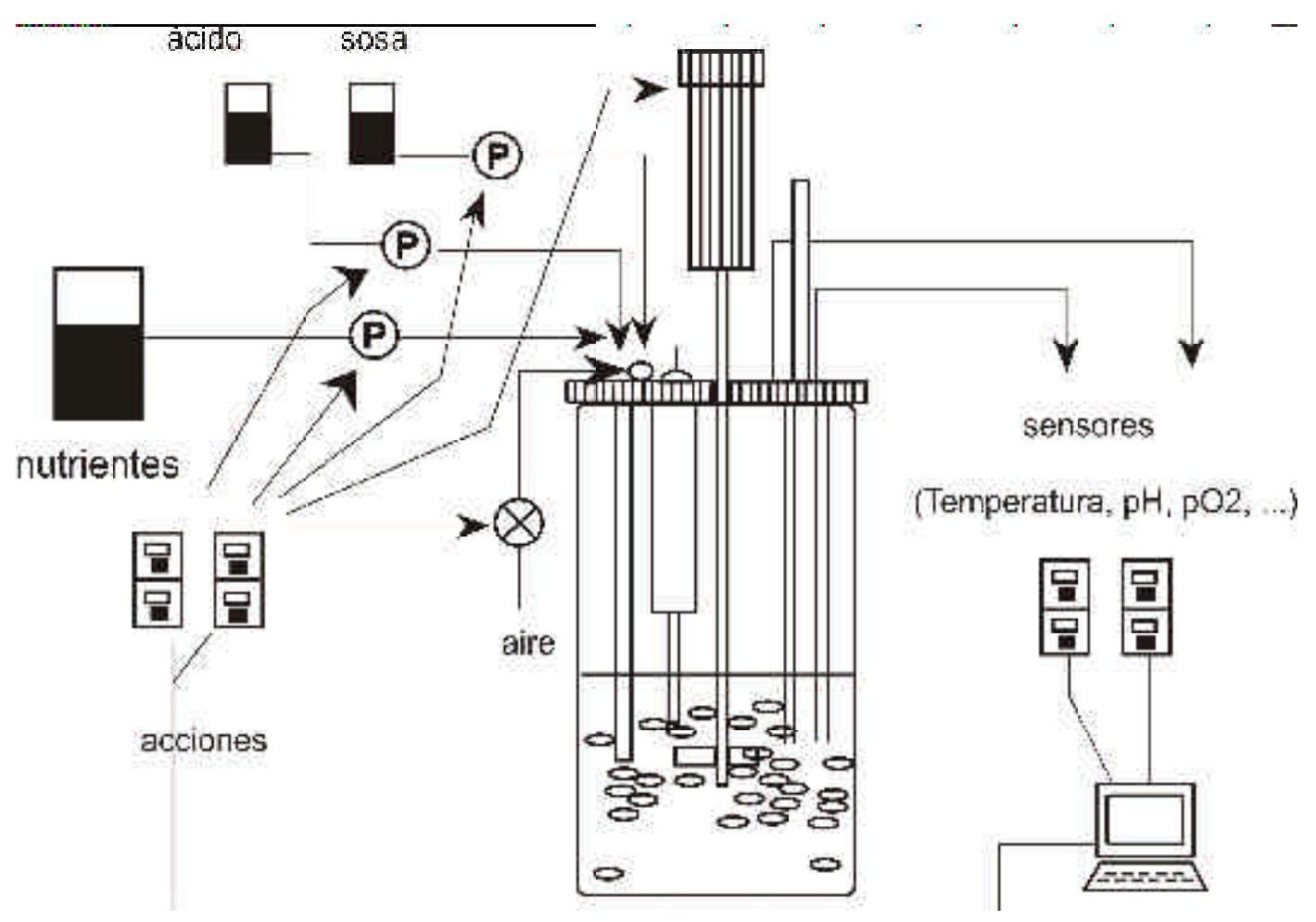

Figura 1. Bioreactor

proceso. Entre estas dos opciones existen múltiples posibilidades caracterizadas por diferentes precios, volúmenes, tecnologías, modos de funcionamiento (discontinuo, fed batch, continuo, cascada), etc. (Monte et al., 2003), (Bailey, 1986). La elección depende de los recursos económicos disponibles y del interés por desarrollar una tecnología propia. A continuación, se expone una breve descripción del proceso de fermentación alcohólica diseñado en la Plataforma de Predesarrollo en Biotecnología (PPB) en Dijon, Francia.

\section{Proceso de fermentación alcohólica a partir de melazas: Resultados de pruebas piloto en volúmenes de $\mathbf{1 0 0}$ litros}

Desde hace varios años, existe el interés (Boudarel, 1984) por diseñar tecnologías de bajo costo que permitan producir bioetanol a partir de desechos o subproductos de origen agrícola; es así que se construyó una instalación piloto de fermentación de 100 litros, acoplada a una unidad de destilación. El objetivo fue realizar estudios de factibilidad y prueba para la obtención de etanol a partir de melazas. La instalación permite realizar la fermentación en modo continuo y en condiciones no estériles, manteniendo concentraciones elevadas de levaduras. El esquema de la instalación se presenta en la figura 2. 


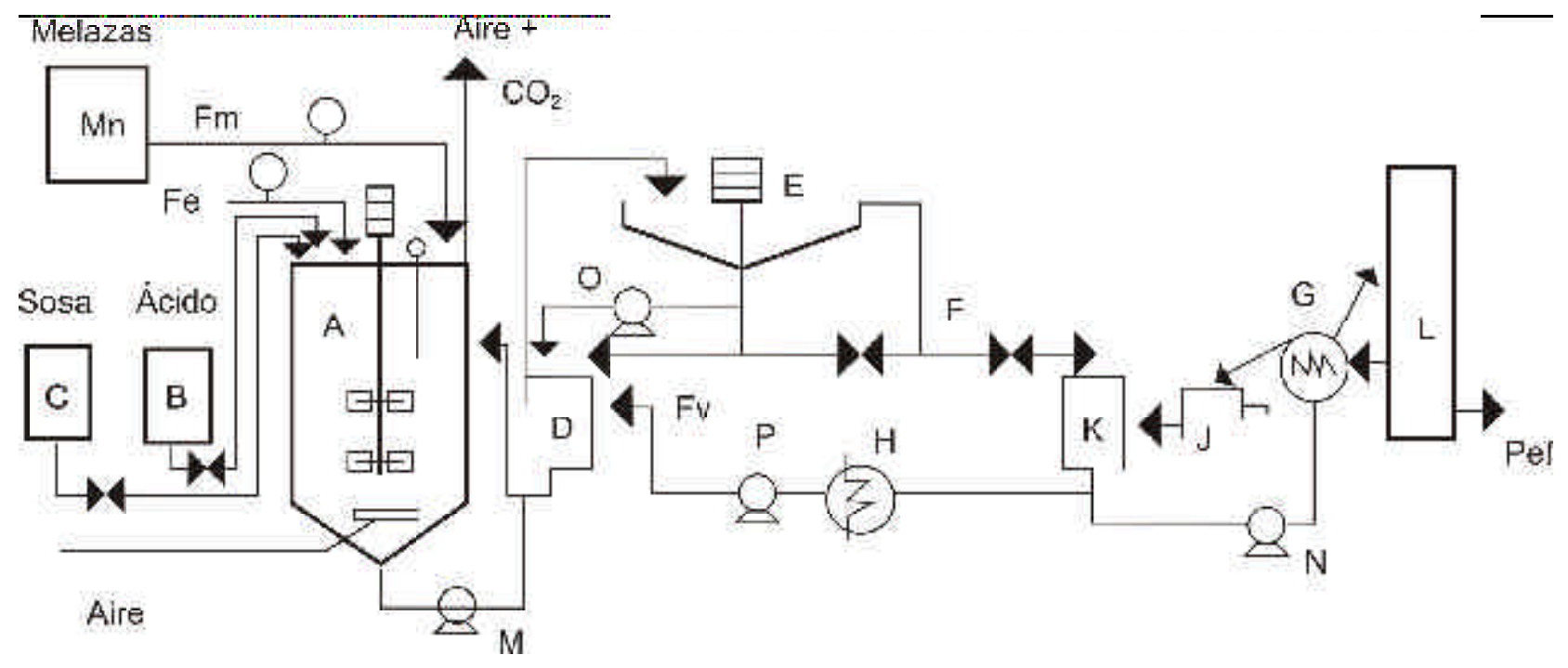

Figura 2. Instalación piloto 100 litros

A: $\quad$ Bioreactor de fermentación.

B y C: Recipientes de almacenamiento de ácido y de base respectivamente.

D Recipiente de recolección de levaduras y vinasas para el reciclaje.

E: Decantador.

Mn: Recipiente de almacenamiento de melazas.

F: Válvulas automáticas.

G: Intercambiador de calor para el calentamiento del caldo de fermentación.

$\mathrm{H}$ : Intercambiador de calor para el enfriamiento de vinasas.

J: $\quad$ Recipiente de recolección de vinasas.

K: $\quad$ Recipiente de recolección del caldo de fermentación.

L: Columna de destilación.

M: Bomba para la crema de levaduras.

$\mathrm{N}$ : Bomba de alimentación a la columna de destilación.

O: $\quad$ Bomba de reciclaje de levaduras.

P: $\quad$ Bomba para el reciclaje de vinasas.

Fm: Flujo de melazas.

Fe: Flujo de agua.

Fair: Flujo de aire.

CO2: Flujo de dióxido de carbono.
Fv: $\quad$ Flujo de vinasas.

Pef: Flujo de etanol.

La instalación integra cuatro subsistemas:

Reserva de melazas (Mn): Recipiente de forma cilíndrica dotado de un sistema de agitación y de un sistema de calentamiento. Una balanza mide la pérdida de peso, permitiendo así calcular la cantidad de melazas que se envía al bioreactor

Bioreactor $(A)$ : Recipiente de forma cilíndrica, en acero inoxidable, equipado con sistemas de medida [temperatura, $\mathrm{pH}$, volumen], de entrada (para las melazas (Fm), agua (Fe), vinasas, ácido y sosa)]; y de salida (para el cultivo fermentado, para la evacuación del aire, para la evacuación del dióxido de carbono y evacuación de las levaduras en exceso). Un motor permite agitar el líquido evitando así la formación de gradientes de concentración y temperatura.

Decantador (E): Un recipiente de forma cónica que permite la recuperación en con- 
tinuo de las levaduras para concentrarlas (Bidault, 1985). El medio de cultivo fermentado se evacúa y luego se recupera en un depósito de recolección, para ser enviado a la columna de destilación. A medida que se requiere, las levaduras se reciclan al birreactor.

Columna de destilación ( $L$ ): La columna de destilación permite separar, por evaporación, el etanol del cultivo de fermentación. Las vinasas, que resultan de la destilación, se reciclan para consumir el azúcar residual.

En la tabla 1 se presentan resultados (promedio) de ensayos con la unidad piloto de 100 litros, y se observa que el tiempo de fermentación varía entre 8 y 25 horas dependiendo del modo de funcionamiento. En el caso de operar el proceso en discontinuo, el tiempo de fermentación aumenta, debido a la necesidad de producir levadura en cantidad suficiente para transformar el azúcar de las melazas. Sin embargo, se logra obtener una concentración de etanol mayor que en otros modos de operación. La concentración de etanol raramente sobrepasa el límite de $9^{\circ}$ $\mathrm{GL}^{2}$. Para lograr estos resultados se utilizó el algoritmo de optimización secuencial Simplex modificado (Nedler and Mead, 1965), supervisado por un sistema experto (Vázquez, 1993).

$2{ }^{\circ} \mathrm{GL}$ significa Grados Guy Lussac. Un grado GL equivale a 12 litros de alcohol por cada 100 litros de medio de cultivo.
Al comparar estos resultados con ensayos de otros sistemas de fermentación se observa lo siguiente:

En sistemas que funcionan en modo discontinuo (Miniac, 1984) se logran obtener concentraciones finales de alcohol entre $8 \mathrm{y}$ $12^{\circ} \mathrm{GL}$ y rendimientos estequiométricos entre 80 y $90 \%$. En modo discontinuo la productividad es baja, entre 1 y $2.5 \mathrm{~g} / \mathrm{h}$. La productividad aumenta en función de la tasa de crecimiento de levaduras, pero disminuye por la inhibición de azúcar y del mismo alcohol). Las cifras obtenidas con nuestra unidad piloto de 100 litros son semejantes.

En ensayos con la unidad piloto de 100 litros en modo continuo, resulta una menor concentración de etanol; sin embargo, en el caso de reciclaje de vinasas los resultados son muy similares a ensayos realizados en otros laboratorios (Tabla 2).

Antes de concluir acerca de la factibilidad de esta propuesta, es importante considerar que la unidad de 100 litros funciona en un ambiente no estéril, y que otros microorganismos compiten por el azúcar, disminuyendo la productividad, lo cual podría parecer una restricción importante; sin embargo, en condiciones no estériles el costo de producción se reduce considerablemente porque no se requiere consumo de energía para esterilizar y eliminar los contaminantes.

Tabla 1. Resultados de ensayos con la unidad piloto de 100 litros

\begin{tabular}{|c|c|c|c|}
\hline Proceso & $\begin{array}{l}\text { Funcionamiento } \\
\text { en modo discontinuo }\end{array}$ & $\begin{array}{l}\text { Funcionamiento en modo } \\
\text { continuo con reciclaje de levaduras }\end{array}$ & $\begin{array}{l}\text { Funcionamiento en modo } \\
\text { continuo con reciclaje de vinasas }\end{array}$ \\
\hline $\begin{array}{l}\text { Concentración de } \\
\text { levaduras g } \Lambda\end{array}$ & $5 \mathrm{~g} /$ & $10-12 \mathrm{~g} /$ & $10-12 \mathrm{~g} /$ \\
\hline $\begin{array}{c}\text { Concentración en } \\
\text { Etanol }^{\circ} \mathrm{GL}^{2}\end{array}$ & $8^{\circ} \mathrm{GL}$ & $4^{\circ} \mathrm{GL}$ & $4-6^{\circ} \mathrm{GL}$ \\
\hline $\begin{array}{l}\text { Tiempo de } \\
\text { Fermentación en } \\
\text { horas }\end{array}$ & $25 \mathrm{~h}$ & $17 \mathrm{~h}$ (tiempo de residencia en el & $8 \mathrm{~h}$ (tiempo de residencia en el \\
\hline
\end{tabular}


Fermentación alcohólica: Una opción para la producción de energía renovable a partir de ...

Tabla 2. Resultados de fermentación alcohólica a partir de melazas

\begin{tabular}{cccc}
\hline Proceso & $\begin{array}{c}\text { Fermentación en } \\
\text { cascada } \\
\text { (Arlie et al., 1984) }\end{array}$ & $\begin{array}{c}\text { Fermentación en modo } \\
\text { continuo con reciclaje } \\
\text { (Miniac, 1984) }\end{array}$ & $\begin{array}{c}\text { Fermentación en modo } \\
\text { continuo con reciclaje de } \\
\text { Vinasas (Miniac, 1984) }\end{array}$ \\
\hline $\begin{array}{c}\text { \% del rendimiento respecto } \\
\text { al rendimiento teórico } \\
\text { Concentración en } \\
\text { levaduras (g } / \text { ) }\end{array}$ & $90-94 \%$ & $90-93 \%$ & $91-95 \%$ \\
$\begin{array}{c}\text { Concentración en } \\
\text { etanol }\left({ }^{\circ} \mathrm{G} \text { L) }\right.\end{array}$ & $15-20$ & - & 8 \\
$\begin{array}{c}\text { Tiempo de } \\
\text { fermentación en horas }\end{array}$ & $8.5-9.5$ & $7-8$ & $4-6$ \\
\hline
\end{tabular}

La esterilización requiere grandes cantidades de energía y su uso reduciría el interés de producir etanol por esta vía.

El uso de la unidad piloto para la producción de etanol a partir de otros desechos o productos agrícolas (azúcar de caña, almidón de trigo, almidón de maíz, papa, sorgo, yuca o cualquier producto de origen vegetal) no debe presentar problemas en la medida en que la materia pueda presentarse en forma líquida, contenga azúcares transformables y no presente substancias inhibidoras para el funcionamiento de la levadura.

\section{Perspectivas y conclusiones}

La biotecnología ofrece diversas opciones para la generación de energías renovables. Dentro de las ventajas más importantes encontramos su carácter sustentable, la garantía de mantener la seguridad y diversidad del suministro energético y la posibilidad de obtener servicios de energía sin impacto ambiental. Una de estas energías renovables, obtenidas por vía biotecnológica, es el bioetanol. Producto que presenta en varios países un interés creciente, pues su uso es posible como carburante de sistemas de transporte (Greencarcongress, 2006).
No obstante, diversos análisis económicos muestran que en un ambiente competitivo, la producción de bioetanol está en desventaja por los costos de obtención de materias primas y el consumo de energía para separar el etanol por destilación. Por ejemplo, un estudio de costos de plantas de producción de bioetanol indica que un 40 a $60 \%$ de los costos dependen de la materia prima; entre un $10 \mathrm{y}$ $16 \%$ dependen del consumo de energía, y entre un 14 y 20\%, son costos de capital (Schulze and Kreen, 2006). Con relación al consumo de energía, existe la posibilidad de ahorrar ésta usando sistemas de destilación que usan la energía solar (Jorapur et al., 1991), otra opción es evitar el uso de energía para esterilizar el substrato como se propone en el presente trabajo.

A pesar de las desventajas de tipo económico, existen otras perspectivas, entre ellas la de proteger nuestros recursos y reducir el impacto ambiental (Sudarsan et al., 2006). Desde ese punto de vista, el bioetanol presenta ventajas, pues su uso en vehículos de transporte reduciría de manera importante las emisiones de $\mathrm{SO} 2, \mathrm{CO} 2$, residuos de hidrocarburos y, al mismo tiempo, permitiría una reducción del efecto invernadero [si un litro de gasolina se remplaza por un litro de 
bioetanol, se evitaría el 75\% de emisiones de gas con efecto de invernadero](Marleix, 2004). Aunque esta ventaja hay que tomarla con reserva, pues resultados con el uso de modelos de simulación, muestran que el uso de etanol a gran escala no mejoraría la calidad del aire en forma significativa e incluso, concluyen que su uso masivo causaría más problemas en el ambiente y en la salud (Jacobson, 2007).

Cabe señalar que la producción de etanol por fermentación es una tecnología limpia, relativamente simple y fácil de desarrollar. $\mathrm{Su}$ producción en bioreactor, aunque compleja desde el punto de vista bioquímico, no requiere de conocimientos especializados para su realización. Sin embargo, no hay que olvidar la importancia de contar con cepas de levadura adecuadas, por ejemplo, cepas floculantes, con resistencia a altas concentraciones de etanol y resistencia a las bacterias. En México, existen diversos centros de investigación como la UNAM, la UAM y el IPN con personal altamente capacitado en la selección de microorganismos, para ellos no representaría gran problema la obtención de cepas de levaduras apropiadas.

Para concluir, es importante puntualizar que en este trabajo se propone la producción de etanol a partir de desechos, subproductos, coproductos y de productos agrícolas de baja demanda, con el objeto de añadir valor agregado a éstos, generar nuevos empleos para mitigar la pobreza de numerosos grupos de campesinos mexicanos y diversificar la producción de energía, favoreciendo el desarrollo sustentable e-n beneficio directo de los habitantes en zonas rurales.

Por supuesto que es importante considerar otros aspectos a nivel global, con el fin de evitar otros problemas derivados de perspectivas unilaterales como la de aumentar sólo la "eficiencia económica" (Sarukhán,
2007), sin considerar aspectos sociales y ambientales. Por ejemplo, la producción masiva de cultivos destinados al etanol puede provocar problemas de deforestación o reducir alimentos provenientes de cereales como el trigo o el maíz. La deforestación por su parte, provocaría una reducción importante de la diversidad biológica, así como una disminución de las funciones de regulación de los recursos acuíferos. Los fertilizantes y pesticidas usados para lograr el cultivo intensivo, además de contaminar suelos y aguas, serían también una causa más de las emisiones de gases contaminantes en forma de dióxido de nitrógeno y metano (Cahier Francais, 2007). La competencia entre la producción de cereales destinados a la energía y los reservados a la alimentación provocaría un aumento desmedido de precios en detrimento de la población. Sin considerar, en el caso de uso de cereales OGM, la incertidumbre que provocaría su liberación en el ambiente.

\section{Agradecimientos}

Héctor Javier Vázquez agradece al Prof. Henri Blachère y al Dr. Alain Durand el gran apoyo para realizar este trabajo durante diferentes estancias en la Estación de Microbiología y en la Plataforma de Predesarrollo en Biotecnología (PPB). El Profesor Blachère, uno de los principales pioneros en el desarrollo de la Biotecnología en Francia, fue director de la Estación de Microbiología, hoy PPB del Instituto Nacional de Investigaciones Agronómicas (INRA), Profesor del Instituto Nacional de Agronomía, Paris Grignon, (INA PG) Francia y fundador de las empresas de fabricación de equipo de fermentación Biolafitte e Inceltech. El Dr. Durand es, hoy en día, el director de la PPB. El autor también agradece a la Región Bourgogne, Francia, quien a través de la Asociación "BOURGOGNE TECHNOLOGIES

A.R.D.T.", le otorgó el apoyo financiero 
Fermentación alcóholica: Una opción para la producción de energía renovable a partir de ...

(1989-1991) para el desarrollo de un Sistema Experto destinado al control y optimización de la instalación Piloto 100 l. También, agradece a la UAM Azcapotzalco por los permisos y licencias, sin goce de sueldo, otorgadas para realizar este proyecto. $\mathrm{Fi}$ nalmente, agradecemos al Prof. Jaime Grabinsky Steider del Departamento de Ciencias Básicas e Ingeniería, UAM Azcapotzalco y a la Lic. Mitzi Pérez Alcázar de la División CSH, UAM Xochimilco, su apoyo y sugerencias para mejorar la lectura de éste documento.

\section{Referencias}

Almeida C., Esteves B. (2006). Nouveaux Défis pour les Biocarburants Brésiliens. Revista Biofutur, No. 269, Septembre, pp. 32-36.

Agencia Internacional de la Energía (2006). http://www.iea.org

Arlie J. P., Ballerini D., Nativel F.(1984). Les Procédés Modernes de Fabri ca tion de l'éthanol de Fermentation. Revista de l'Institut Français du Pétrole, Vol. 39, No. 6. Noviembre-Diciembre, pp. 781-805.

Bailey J.E. (1986). Biochemical Engineering Fundamentals. 2a. ed. Mc Graw Hill.

Bidault C. (1985). La Fermen ta tion Alcoolique avec Recyclage: Mise au point d'un decanteur. Mémoire d’Ingénieur. ENGREF.

Boudarel M.J.(1984). Contribution á l'étude de la Fermen ta tion Alcoolique á partir de jus de Betteraves avec. Saccharomyces cerevisiae. Thèse de Doctorat. Université de Dijon, Francia.

Cahier Francais (2007). Instru ments y Politiques. L'énergie au XXIé siècle: un défi environnemental majeur. Cahier francais 337. Ladocumen ta tion Francaise, Mars-Avril, pp. 69-75.

Coalición Americana por el Etanol (2006), http://www.ethanol.org/
Environemental Protec tion Agency (2007), http://www.epa.gov/mtbe/faq.html\# concerns

Greencarcongress (2006), http://www.greencarcongress.com/bio diesel/

Instituto Francés del Petróleo (2006), http://www.ifp.fr/IFP/en/aa.htm

Jacobson M. (2007). Effects of Ethanol (E85) Versus Gasoline Vehicles on Cancer and Mortality in the United States.Environ. Sci. Technol., Vol. 41, pp. 4150-4157.

Jorapur R.M., Rajvanshi A. (1991). Alcohol Distillation by Solar Energy. Solar World Congress Proceedings. Pergamon Press, Vol. I, Part II, pp. 772-777.

Lemire S., Ashley D., Olaya P. Romieu I., Welch S., Meneses F. and Hernández M. (2004). Environmental Esposure of Commuters in Mexico City to volatile Organic Compounds as Assessed by Blood Concentrations, 1998. Revista Salud Pública de México, Vol. 46, No.1, pp. 5-7, Enero-Febrero.

Make your own fuel (2006). http://running_on_alcohol.tripod.com/ Marleix M.A.(2004). Rapport 1622 d'information, Asamblea Nacional de Francia. Sur Les Biocarburants. Article 146, Règlement Commis sion des Finances, de L'Économie Générale. Asamblea Nacional de Francia, Francia, Mayo 26.

Maurice J. (2007). Demande d'énergie et de Matières Premières: Les Limites Approchent. Revue ESPRIT, Francia, Juin, number 335, pp. 45-53.

Miniac M.N.(1984). Gain de Productivité d'Ethanol en Fermentation Alcooloque des Produits de Sucrerie (Mélasses et Egouts). Idustries. Alimentaires. Agricoles, 102, pp. 971.

Monte A.R., Rigo M., Joekes I. (2003). Ethanol Fermentation of a Diluted Molasses Medium by Saccharomyces 
cerevisiae immobilized on chrysotile Braz. Archives of Biology and Techno$\log y$, Vol.46, No. 4, Curitiba, Diciembre.

Nava V. and Morales M. (2006). Degradation of Metyl tert-Butyl Ether (MTBE) by Pseudomonas Strains. The Second International Meeting on Envi ron mental Biotech nology and Engineering, Mexico City, Mexico, Septiembre.

Nelder J.A. and Mead R.(1965). A Simplex Method for Function Minimization, Computer J., 7, pp. 308-313.

Sarukhán J. (2007). Los conceptos de eficiencia tendrán que cambiar. Entrevista, Letras Libres, México, DF.

Secretaría de Energía (2005), http://www.energia.gob.mx/work/res ources/LocalContent/1808/1/
Sudarsan, K.G., Anupama M.P. (2006). The Relevance of Biofuels. Current Science, Vol. 90, No. 6, 25 march.

Schulze T., Kreen W. (2006). Pötschacher P. Planning and Construction of $a$ Bioethanol Plant. Association of Cereal Research. http://www.agfdt.de

Vázquez H.J. (1993). Contribution des Systémes Experts á la Conduite et á l'Optimisation d'un Procédé de Fermentation Alcoolique. Thèse de Doctorat de 1’Universidad de Tecnología de Compiègne, Francia.

Verdesio J. (2003). Políticas públicas para la difusión de las nuevas energías renovables. Coloquio: “Energía, reformas estructurales y desarrollo en América Latina". Brasil. Http://www.unb.br/fav/renova/INDE X.html

\section{Semblanza de los autores}

Héctor Javier Vázquez. Realizó la licenciatura en ingeniería bioquímica en la Escuela Nacional de Ciencias Biológicas del IPN. La Universidad de Pennsylvania le otorgó los grados de maestro en ciencias en bioingeniería e ingeniería en sistemas. En la Universidad de Tecnología de Compiègne (UTC) en Francia, logró el grado de doctorado en ingeniería de procesos. La Escuela Nacional de Estadística y Análisis de b Información (ENSAI), de l'INSEE Francia, le concedió el grado de maestro en ciencias en estadística. Durante su estancia en Francia, colaboró con el Instituto Nacional de Investigaciones Agronómicas (INRA), en Dijón, donde trabajó en la construcción y automatización de sistemas de fermentación. En México, es profesor investigador en el Departamento de Sistemas de la Universidad Autónoma Metropolitana, Unidad Azcapotzalco y responsable del Cuerpo Académico Análisis y Manejo de Información.

Ophélia Dacosta. Realizó sus estudios de licenciatura y posgrado en la Universidad de Lisboa, Portugal, la Universidad de Borgoña y en la Sorbona, Francia. Desde 1993, es consultora experta en Portugal, Francia y en la Unión Europea. 